\title{
Comparison of Self-Etching Adhesives and Etch-and-Rinse Adhesives on the Failure Rate of Posterior Composite Resin Restorations: A Systematic Review and Meta-Analysis
}

\author{
Basílio Rodrigues Vieira ${ }^{1}$ Eugênia Lívia de Andrade Dantas ${ }^{1}$ Yuri Wanderley Cavalcanti ${ }^{2}$ \\ Bianca Marques Santiago ${ }^{2}$ Frederico Barbosa de Sousa ${ }^{3}$ \\ ${ }^{1}$ Graduate Program in Dentistry, Health Sciences Center, Federal \\ University of Paraíba, João Pessoa, Paraiba, Brazil \\ 2 Department of Clinical and Social Dentistry, Health Sciences Center, \\ Federal University of Paraíba, João Pessoa, Paraiba, Brazil \\ ${ }^{3}$ Department of Morphology, Health Sciences Center, Federal \\ University of Paraíba, João Pessoa, Paraiba, Brazil

\begin{abstract}
Address for correspondence Basilio Rodrigues Vieira, MD, Graduate Program in Dentistry, Health Sciences Center, Federal University of Paraiba, 58051-900 João Pessoa, Brazil
\end{abstract} \\ (e-mail: basilio_451@hotmail.com). \\ Eur J Dent 2022;16:258-265.
}
Abstract
Keywords
- dentine adhesives
- resin composite
- dental restoration
- failures
- clinical studies
- meta-analysis

The aim of this study was to perform a systematic review with meta-analysis on the comparison of self-etching adhesives and etch-and-rinse adhesives with respect to the failure rate of posterior composite resin restorations. The study protocol was registered in PROSPERO (CRD42017078015), following PRISMA recommendations and PICO search strategy. Literature search was performed in the following databases: MEDLINE, ISI Web of Science, LILACS, SCOPUS, and Cochrane Library through July 2021. Six studies from five randomized clinical trials were included in the qualitative synthesis. The funnel plot detected important bias (all studies out of the funnel area). The metaanalysis showed a positive summary Cohen H effect size of 0.406 ( $95 \% \mathrm{Cl}: 0.100 ; 0.713$, $p=0.009$ ), favoring etch-and-rinse adhesives. The total number of failures (including restorations that required replacement and those that did not require replacement) were attributed to either marginal adaptation (five studies) or marginal staining (one study). A very low certainty of the evidence was obtained through GRADE analysis. In conclusion, current available evidence indicates that etch-and rinse adhesives performed better (with a low effect size) than self-etching adhesives in terms of failure rates in posterior composite restorations.

\section{Introduction}

Dentine adhesives, which have undergone substantial changes over the last 20 years, are classified into two techniques: self-etch or etch-and-rinse. ${ }^{1}$ Etch-and-rinse, the first to be introduced, is the technique that results in the deepest hybrid layer in enamel. ${ }^{2}$ Because of the higher

published online November 22, 2021
DOI https://doi.org/ 10.1055/s-0041-1736332. ISSN 1305-7456. number of steps and stronger effect of the etching procedure on dentine substrate, etch-and-rinse technique requires a longer clinical application time, results in increased postoperative sensitivity, and is the more sensitive to failure. ${ }^{2}$

The shorter application time and decreased postoperative sensitivity favors the choice for the self-etch, ${ }^{3}$ but their

\section{(C) 2021. The Author(s).}

This is an open access article published by Thieme under the terms of the Creative Commons Attribution License, permitting unrestricted use, distribution, and reproduction so long as the original work is properly cited. (https://creativecommons.org/licenses/by/4.0/)

Thieme Medical and Scientific Publishers Pvt. Ltd., A-12, 2nd Floor, Sector 2, Noida-201301 UP, India 
thinner hybrid layer raises concern on whether the durability of the restoration is reduced or not. ${ }^{4}$ At the other hand, the thinner dentine hybrid layer theoretically provides less substrate to be degraded by chemical (both hydrolysis and enzymatic) and mechanical factors.

Adhesive composite restorations in posterior teeth are currently the first choice for direct restorations in posterior teeth, ${ }^{5,6}$ and their popularity is expected to increase with current prevailing conservative philosophy in the restorative treatment where minimal hard dental tissue removal is recommended. Self-etch technique is in line with such a philosophy, and short duration clinical studies have reported similarities between self-etch and etch-and-rinse techniques with respect to clinical outcomes. ${ }^{7-9}$ However, relying mostly on statistical analysis restricted to $p$-values (statistical significance), the individual scientific contribution of those studies to the choice of the appropriate dentine adhesive technique is questionable. ${ }^{10,11}$

To the best of our knowledge, there are no systematic reviews with meta-analysis comparing self-etch and etchand-rinse techniques for dentine adhesives in posterior composite restorations. Filling the gap in such an important topic in clinical Dentistry could provide an important contribution to the restorative dental practice with maximum preservation of tooth structure.

Therefore, the aim of this study was to perform a systematic review with meta-analysis on the comparison of selfetching adhesives and etch-and-rinse adhesives on the failure rate of posterior composite resin restorations.

\section{Methods}

\section{Focused Question}

This systematic review was aimed at answering the following research question: do composite resin restorations in posterior teeth performed with either self-etch or etch-and-rinse techniques differ in the clinical failure rate?

This review followed the PRISMA guidelines, ${ }^{12}$ and its protocol was registered in the International Prospective Register of Systematic Reviews (PROSPERO) under the number CRD42017078015.

\section{Search Strategy}

The literature search included studies published through July 2021, and it was undertaken by two independent researchers in the following databases: MEDLINE (PubMed), ISI Web of Science, LILACS, SCOPUS, and Cochrane Library, in addition to searches in grey literature (Google Scholar and manual search in the list of references of included studies).

Search strategies for the literature are based on PICO acronym. ${ }^{13}$ A combination of MeSH terms, keywords, and related terms was used in the systematic literature search in conjunction with Boolean operators "AND" and "OR" (-Table 1).

\section{Screening and Study Selection}

Duplicate removal was undertaken by two independent examiners (B.R.V. and E.L.A.D.), using Mendeley software (version 1.5.2 for Windows). Article selection for inclusion was based on the evaluation of titles, abstracts (step1), and then evaluation of full texts (step 2). Only randomized clinical trials, controlled clinical trials, and nonrandomized controlled prospective studies were selected for this systematic review. Observational studies, case reports, cases series, in vitro studies, literature review, editorials, and letters to the editor were excluded.

Full analysis of selected articles was undertaken based on the following PICO terms ${ }^{13}$ : Population represented by posterior permanent teeth with Class I or Class II resin composite restorations due to caries, Intervention represented by selfetch adhesives, Control represented by etch-and-rinse (conventional) adhesives, and Outcome represented by failures in restorations that compromise longevity. Disagreements between examiners were solved by consensus. When disagreement persisted, the opinion of a third examiner (B.M.S.) was used.

\section{Data Collection}

Full texts were accessed for validation of eligibility criteria, and the following data were collected: study design, population, group sample, adhesive type, outcome, evaluation criteria, time of follow-up evaluation, statistical analysis, main results, failure rate (marginal staining, marginal adaptation, secondary caries, fractures and retention, and postoperative sensitivity), limitations, and conclusions. This was undertaken independently by two reviewers.

\section{Risk of Bias (Quality Assessment)}

Quality assessment of selected studies was performed individually and independently by two examiners (B.R.V. and E.L. A.D.) using the Cochrane Collaboration Risk of Bias tool, ${ }^{14}$ and the following aspects were analyzed: sequence generation, allocation concealment, blinding of participants and personnel, blinding of outcome assessors, incomplete outcome data, and other sources of bias. Studies were then classified as low, medium, or high risk of bias; those with insufficient information were classified as unclear.

\section{Data Analysis}

From each study, differences between two groups (etch-andrinse as control and self-etching as intervention) were considered. Using data on failure rates (proportions) and sample size per group for each study, we calculated the effect size of difference between proportions using Cohen $\mathrm{H}$ effect size [difference between arcsine transformation of proportions: arcsine $\left.{ }^{*} \operatorname{sqrt}(\mathrm{p} 1)-\operatorname{arcsine}{ }^{*} \operatorname{sqrt}(\mathrm{p} 2)\right]$ and statistical power, following equations described in the literature. ${ }^{15}$ Only failures related to the adhesive were included, which comprised marginal staining, marginal adaptation, secondary caries, fractures and retention, and postoperative sensitivity. The unit restoration with failure was considered as a restoration with one or more failures, so that computation of more than one failure per restoration was excluded. The unit restoration with failure was recorded regardless of the need of restoration replacement. For each group (intervention or control), proportions of restorations with failures were 
Table 1 Search strategies for the literature based on PICO acronym, with the use of Boolean operators and adapted to each database

\begin{tabular}{|c|c|}
\hline Databases & Search strategies \\
\hline PubMed & $\begin{array}{l}(((((((\text { Self-etching adhesives[Title/Abstract]) OR Self etching adhesive[Title/Abstract]) OR All in one adhesive } \\
\text { [Title/Abstract]) OR All in one adhesives[Title/Abstract]) OR One-step adhesive[Title/Abstract])) AND } \\
((((((((((((D e n t i n-b o n d i n g \text { agents[MeSH Terms]]) OR Dentin-bonding agents[Title/Abstract]) OR Agents, Dentin- } \\
\text { Bonding[Title/Abstract]) OR Bonding Agents, Dentin[Title/Abstract]) OR Agents, Dentin Bonding[Title/Abstract]) } \\
\text { OR Dentin Bonding Agents[Title/Abstract]) OR Etch[Title/Abstract]) OR rinse adhesives[Title/Abstract]) OR Etch- } \\
\text { and-rinse[Title/Abstract]) OR Three step adhesive[Title/Abstract]]) OR Three step adhesives[Title/Abstract])))) } \\
\text { AND (((((((Dental Restoration Failure[MeSH Terms]) OR Dental Restoration Failure[Title/Abstract]) OR Failure, } \\
\text { Dental Restoration[Title/Abstract]]) OR Restoration Failure, Dental) OR Restoration Failures, Dental) OR Dental } \\
\text { Restoration Failures) OR Failures, Dental Restoration) }\end{array}$ \\
\hline Scopus & $\begin{array}{l}\text { (TITLE-ABS-KEY(Self-etching adhesives) OR TITLE-ABS-KEY(Self etching adhesive) OR TITLE-ABS-KEY(All in one } \\
\text { adhesive) OR TITLE-ABS-KEY(AII in one adhesives) OR TITLE-ABS-KEY (One-step adhesive)) AND (TITLE-ABS-KEY } \\
\text { (Dentin-bonding agents) OR TITLE-ABS-KEY(Agents, Dentin-Bonding) OR TITLE-ABS-KEY(Bonding Agents, Dentin) } \\
\text { OR TITLE-ABS-KEY(Agents, Dentin Bonding) OR TITLE-ABS-KEY (Etch) TITLE-ABS-KEY(rinse adhesives) OR TITLE- } \\
\text { ABS-KEY(Etch-and-rinse) OR TITLE-ABS-KEY(Three step adhesive) OR TITLE-ABS-KEY(Three step adhesives)) AND } \\
\text { (TITLE-ABS-KEY(Dental Restoration Failure) OR TITLE-ABS-KEY(Failure, Dental Restoration) OR TITLE-ABS-KEY } \\
\text { (Failure, Dental Restoration) OR TITLE-ABS-KEY(Restoration Failures, Dental) OR TITLE-ABS-KEY (Dental } \\
\text { Restoration Failures) OR TITLE-ABS-KEY (Failures, Dental Restoration)) }\end{array}$ \\
\hline Lilacs & 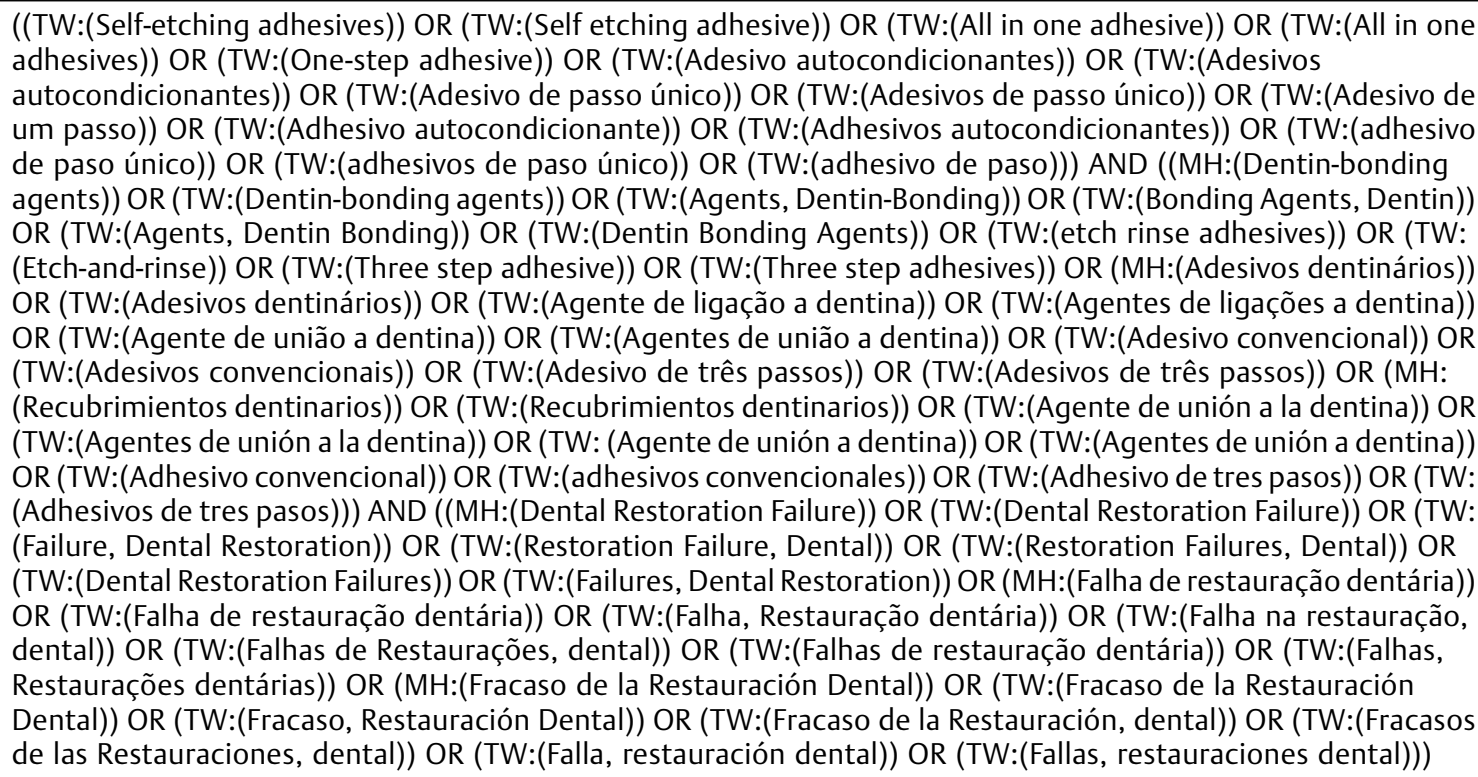 \\
\hline $\begin{array}{l}\text { Web of } \\
\text { Science }\end{array}$ & $\begin{array}{l}\text { TS = (Self-etching adhesives OR Self etching adhesive OR All in one adhesive OR All in one adhesives OR One-step } \\
\text { adhesive) AND TS = (Dentin-bonding agents OR Agents, Dentin-Bonding OR Bonding Agents, Dentin OR Agents, } \\
\text { Dentin Bonding OR Dentin Bonding Agents OR etch rinse adhesives OR Etch-and-rinse OR Three step adhesive OR } \\
\text { Three step adhesives) AND TS = (Dental Restoration Failure OR Failure, Dental Restoration OR Restoration Failure, } \\
\text { Dental Restoration Failures, Dental OR Dental Restoration Failures OR Failures, Dental Restoration) }\end{array}$ \\
\hline
\end{tabular}

computed using the number of restorations with failures divided by the number of restorations.

A two-tailed 5\% significance level was used. The 95\% confidence interval (CI) for Cohen $\mathrm{H}$ was calculated using formula for sampling variance described elsewhere. ${ }^{16}$ Considering that some failure rates in controls were lower than $10 \%$, risk ratio (attributable risk) was not computed because it overestimates the effect size when the proportion of controls is lower than $10 \%{ }^{17}$

Statistical power, whose threshold of $80 \%$ is used to determine whether studies were conclusive (acceptable probability that an effect exists in the population $)^{15}$ or not, was calculated for all studies selected for meta-analysis.
One meta-analysis was performed. Following published statistical procedures, ${ }^{15,16,18}$ we calculated the effect size (Cohen $\mathrm{H}$; es), standard error, sampling variance, individual study weights $(w)$, the weighted effect sizes ( $w^{*}$ es), and the corresponding squared values ( $w 2$ and $w^{*}$ es2). Both Cochran $Q$ test and $I^{2}$ were computed, ${ }^{18}$ and the level of heterogeneity was graded as low (25\%), moderate $(50 \%)$, or high $(75 \%) .{ }^{19}$ The summary outcome was calculated using the fixed effects model when heterogeneity was very low, otherwise the random effects model was used. ${ }^{19}$ The statistical power of the meta-analysis was also computed. ${ }^{20,21}$ A forest plot was prepared using calculated parameters. In addition, bias was also investigated using funnel plots (scatter plots of effect 


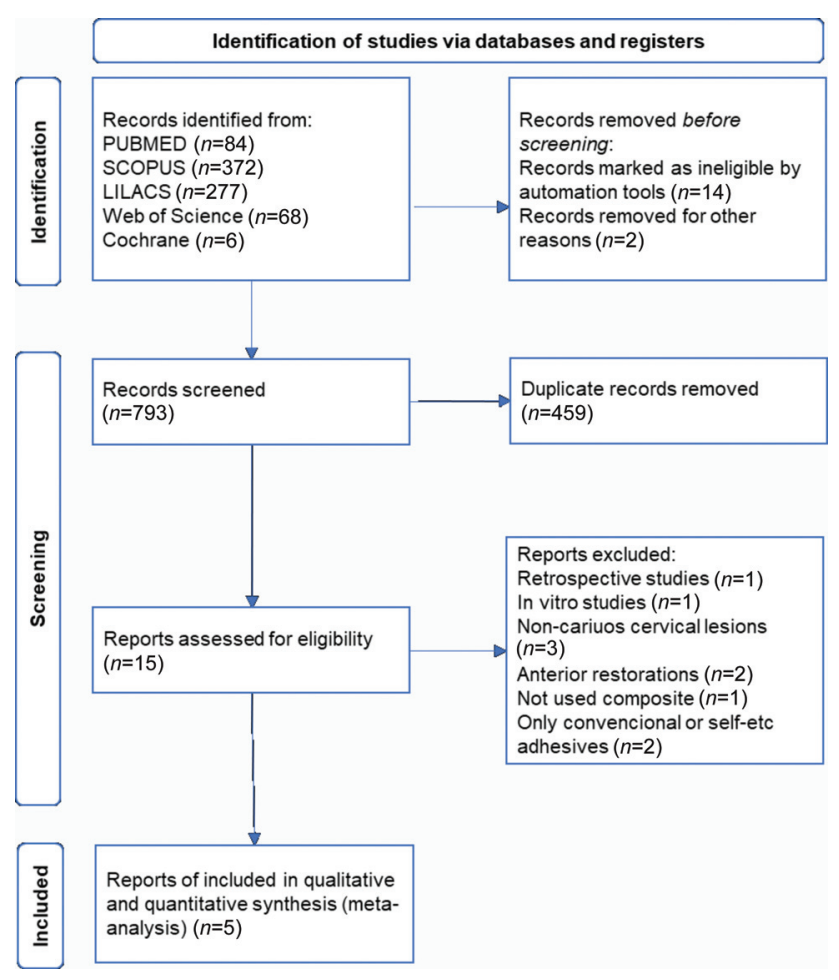

Fig. 1 Flowchart of the steps of the literature search.

sizes in the $\mathrm{X}$ axis against the effect size's standard error in the $\mathrm{Y}$ axis). ${ }^{22}$

\section{Certainty of Evidence}

The certainty of the evidence was assessed through Grades of Recommendations, Assessment, Development and Evaluation (GRADE) approach. The initial ratings followed the recommendations of GRADE group and the certainty of evidenced initiated as high, since this systematic review was performed with randomized clinical trials. The outcome "failure rate of resin restorations" was carefully analyzed for each of the five domains that can lower the certainty: risk of bias, inconsistency, indirectness, imprecision, and publication bias. $^{22}$

\section{Results}

The flowchart and reasons for exclusion of articles are shown in -Fig. 1. A total of 823 articles were recovered, of which 459 were duplicates (removed using the Mendeley software). After careful analysis of titles and abstracts, 15 articles were selected for full text reading, and five articles ${ }^{9,23-26}$ met the inclusion criteria. One article ${ }^{16}$ contained three studies, and one study was excluded because the adhesive (iBond) was not recommended for clinical use by the authors, yielding a total of six studies (five papers with one study each and one paper with two studies) included in the review. The characteristics of the included studies are presented in -Table 2.

All studies compared etch-and-rinse (conventional) and self-etch adhesives with respect to the differences between failure rates of resin composite restorations in posterior teeth (Class I and II). All studies were randomized clinical trials. ${ }^{9,23-26}$

A total of 699 resin composite restorations were analyzed during $2^{23}$ to $8^{9}$ years of follow-up; 342 restorations in the control group (etch-and-rinse adhesive), and 357 in the intervention group (self-etch). Only two studies ${ }^{23,26}$ used rubber dam for moisture control during the restorative procedure.

The following brands of dentine adhesives were reported in the selected studies: Xeno III (Dentsply, Ballaigues, Suíça), Excite (Ivoclar Vivadent, Schaan, Liechtenstein), Prime\&Bond Elect Universal (Dentsply, Milford, United States), Single Bond Universal (3M ESPE, Neuss, Germany), Gluma Bond Universal (Heraeus Kulzer, Germany), One Step Plus (Bisco, Schaumburg, United States), iBond (Heraeus Kulzer, Germany), Clearfil Universal Bond (Kuraray Noritake, Okayama, Japan), Clearfil SE (Kuraray Noritake, Okayama, Japan), Adper Prompt (3M ESPE, St Paul, United States), Allbond Universal (Bisco, Schaumburg, United States), OptiBond XTR (Kerr, Orange, United States) in addition to adhesives modified by the authors.

Generally, the studies used similar criteria of evaluation for failed dental restoration. The United States Public Health Services (USPHS) system was used, with some modifications among studies which did not preclude comparisons: USPHS Ryge system was reported in three articles, ${ }^{9,24,25}$ while another paper $^{23}$ reported the Modified USPHS direct evaluation criteria. Calibrated examiners were reported in all papers. ${ }^{9,23-26}$

From the quality assessment and risk of bias analysis using the Cochrane Collaboration Risk of Bias tool, two studies presented low risk, and the other three presented high risk (-Table 3 ). The main aspects related to high risk were modification of adhesives by authors, ${ }^{25}$ and lack of use of rubber dam for moisture control. ${ }^{9,24,25}$

From the statistical analysis of individual studies, low statistical power was computed for most studies (-Fig. 2). The low power values are accompanied by wide $95 \%$ CIs of the effect size ranging from negative values (favoring the intervention group, self-etch adhesives) to positive values (favoring the control group, etch-and-rinse adhesives), indicating that sample sizes were smaller than required for the relatively large variability.

For each study, a single failure type accounted for the total number of restorations with failure: marginal adaptation in five studies ${ }^{23-26}$ and marginal staining in one study. ${ }^{9}$

Meta-analysis of all selected studies was performed using the random effects model due to the low heterogeneity $\left(I^{2}=16.59 \%\right.$; Cochrane Q test's $p$-value of 0.309$)$ computed for this model. The meta-analysis showed a low summary positive effect size (0.406) with a wide $95 \% \mathrm{CI}(0.100 ; 0.713$; $p=0.0093$ ) and power of $73.91 \%$, favoring etch-and-rinse adhesives (-Fig. 2 ).

The funnel plot detected the presence of important bias (-Fig. 3).

The assessment of the certainty of evidence through GRADE approach revealed a very low certainty of evidence (-Fig. 4). Although the certainty initiated as high through 


\begin{tabular}{|c|c|c|c|c|c|}
\hline 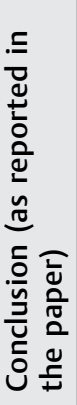 & 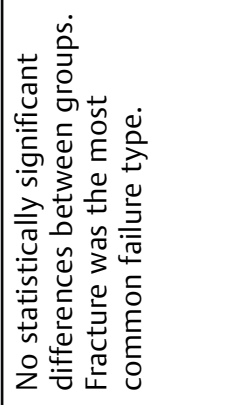 & 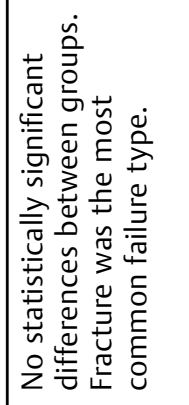 & 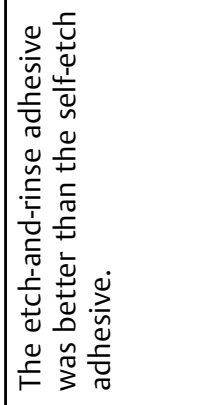 & 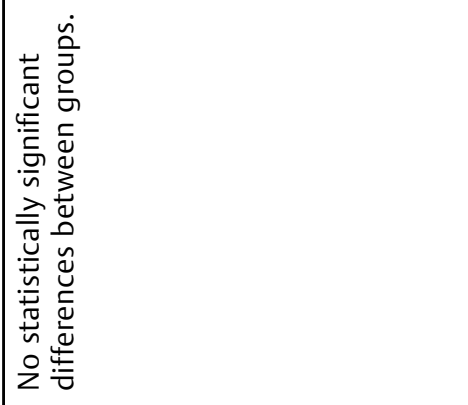 & 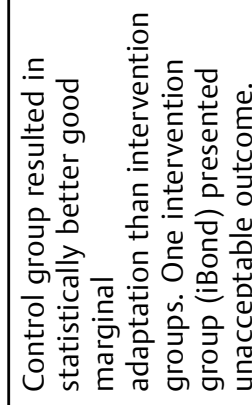 \\
\hline & 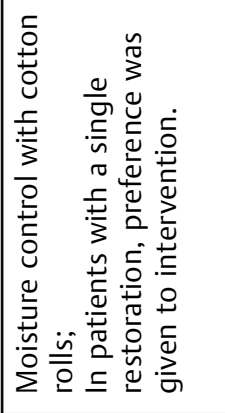 & 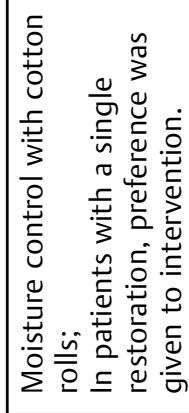 & 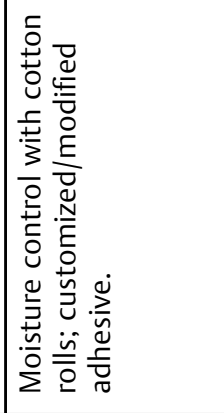 & 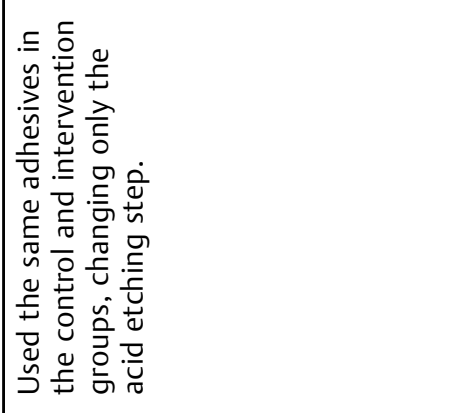 & 1 \\
\hline 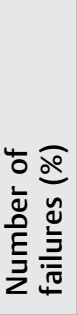 & 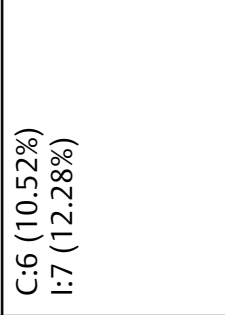 & 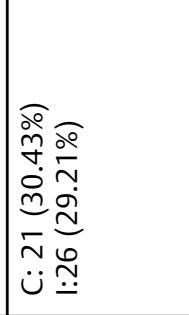 & 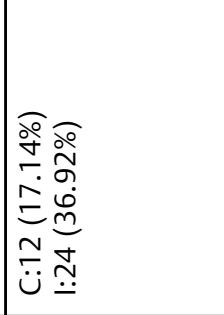 & 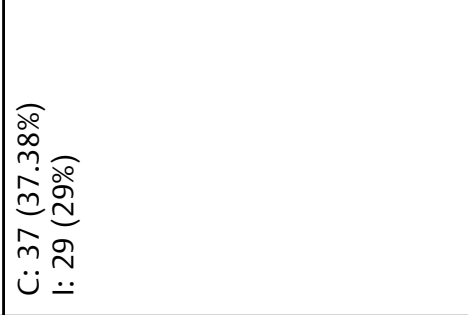 & 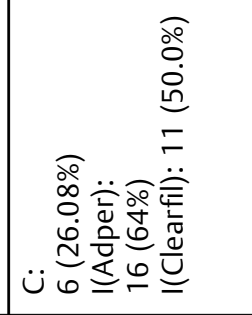 \\
\hline 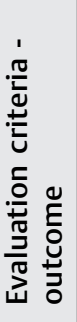 & 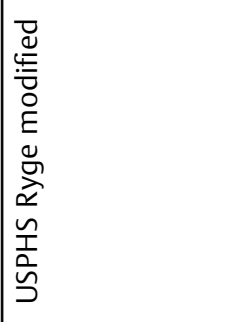 & 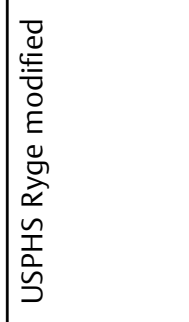 & 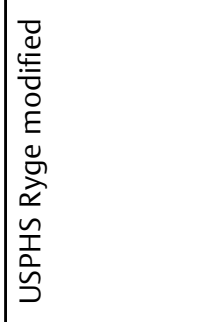 & 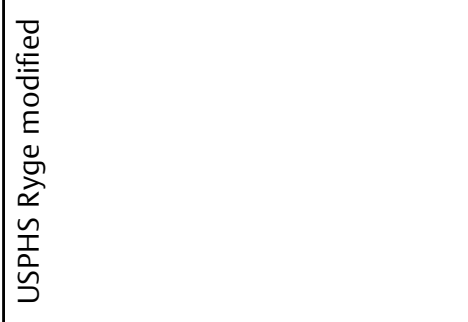 & 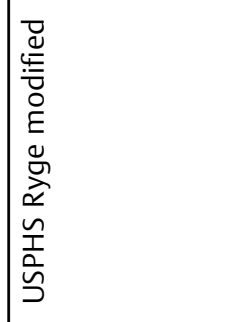 \\
\hline $\begin{array}{l}\text { й } \\
\text { 입 }\end{array}$ & 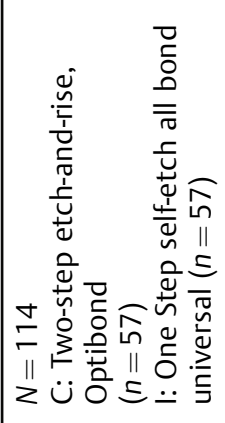 & 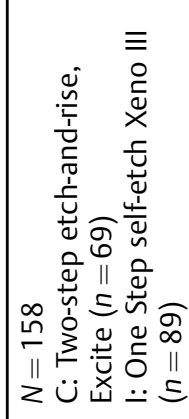 & 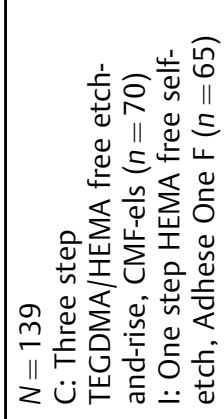 & 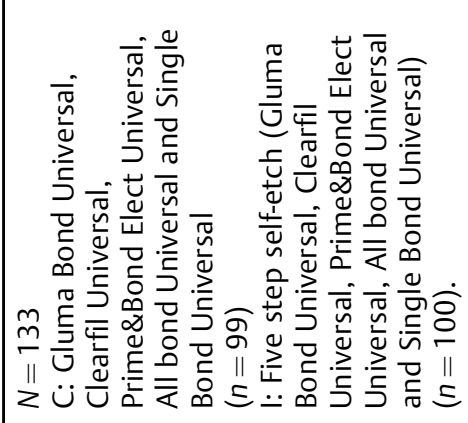 & 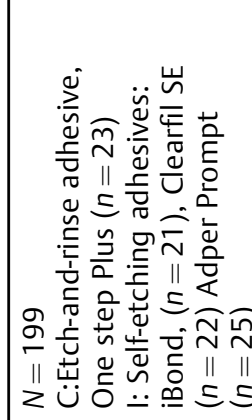 \\
\hline 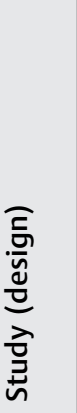 & 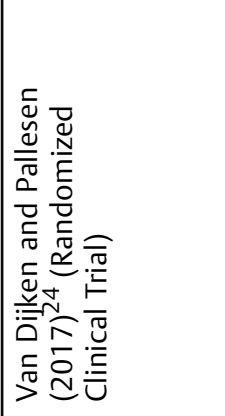 & 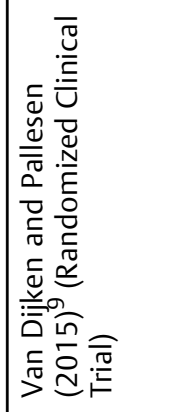 & 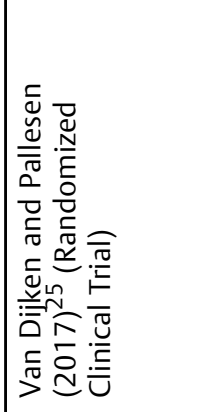 & 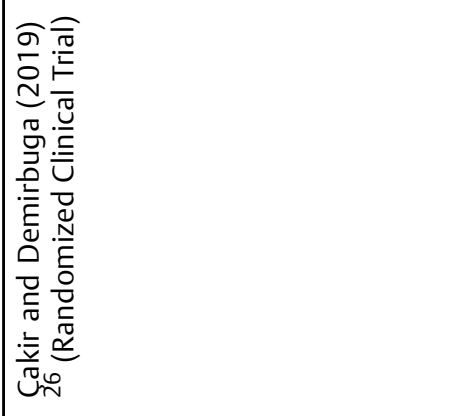 & 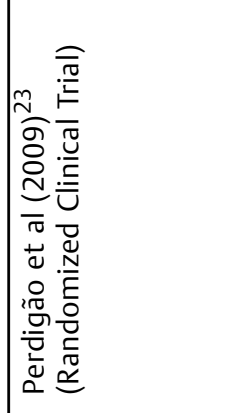 \\
\hline
\end{tabular}


Table 3 Quality assessment and risk of bias according to Cochrane Risk of Bias Tool ${ }^{14}$

\begin{tabular}{|c|c|c|c|c|c|c|c|c|}
\hline Studies & $\begin{array}{l}\text { Sequence } \\
\text { generation }\end{array}$ & $\begin{array}{l}\text { Allocation } \\
\text { Concealment }\end{array}$ & $\begin{array}{l}\text { Blinding of } \\
\text { participants } \\
\text { and personnel }\end{array}$ & $\begin{array}{l}\text { Blinding of } \\
\text { outcome } \\
\text { assessors }\end{array}$ & $\begin{array}{l}\text { Incomplete } \\
\text { outcome } \\
\text { data }\end{array}$ & $\begin{array}{l}\text { Selective } \\
\text { outcome } \\
\text { reporting }\end{array}$ & $\begin{array}{l}\text { Other } \\
\text { sources } \\
\text { of bias }\end{array}$ & Risk of bias \\
\hline $\begin{array}{l}\text { Van Dijken and } \\
\text { Pallesen }(2017)^{24}\end{array}$ & Yes & No & Yes & Yes & Yes & Yes & No & High risk \\
\hline $\begin{array}{l}\text { Van Dijken and } \\
\text { Pallesen }(2015)^{9}\end{array}$ & Yes & No & Unclear & Yes & Yes & Yes & No & High risk \\
\hline $\begin{array}{l}\text { Van Dijken and } \\
\text { Pallesen }(2017)^{25}\end{array}$ & Yes & No & Unclear & Yes & Yes & Yes & No & High risk \\
\hline $\begin{array}{l}\text { Çakir and } \\
\text { Demirbuga } \\
(2019)^{26}\end{array}$ & Unclear & No & Yes & Yes & No & No & No & Low risk \\
\hline $\begin{array}{l}\text { Perdigão } \\
\text { et al }(2009)^{23}\end{array}$ & Unclear & Yes & No & Yes & Yes & No & Yes & Low risk \\
\hline
\end{tabular}

the five studies included in this systematic review, which were randomized controlled clinical trial, the process of detailed ratings across the five domains that can lower the certainty, downgraded this certainty. The critical domains were: (1) Risk of bias, illustrated in - Table 3, that revealed problems related to sequence generation, blinding of participants and operators, incomplete outcome data and selective reporting outcome in the majority of studies, leading to downgrading the certainty of evidence in two levels; (2) Imprecision, observed through the wide CIs. We downgraded the certainty just in one level, since the number of restorations included in metanalyses was above the rule-of-thumb of 400 (200 per group) and also above the optimal information size calculated ( $n=89$ after loss of follow-up); (3) Publication bias, which was suspected analyzing the sample size of each study included, was small, and also confirmed through the funnel plot.

\section{Discussion}

Our review detected six studies that met the inclusion criteria, and the main research question was whether the self-etch adhesives differed from etch-and-rinse adhesives in terms of failure rates of composite resin restoration in posterior permanent teeth. In addition to considering the statistical significance reported in the papers, we further computed effect sizes (intensity of the difference between failure rates), their CIs, and power. The analysis detected that four out of six selected studies presented individually wide CIs, which means that the sample size was smaller than

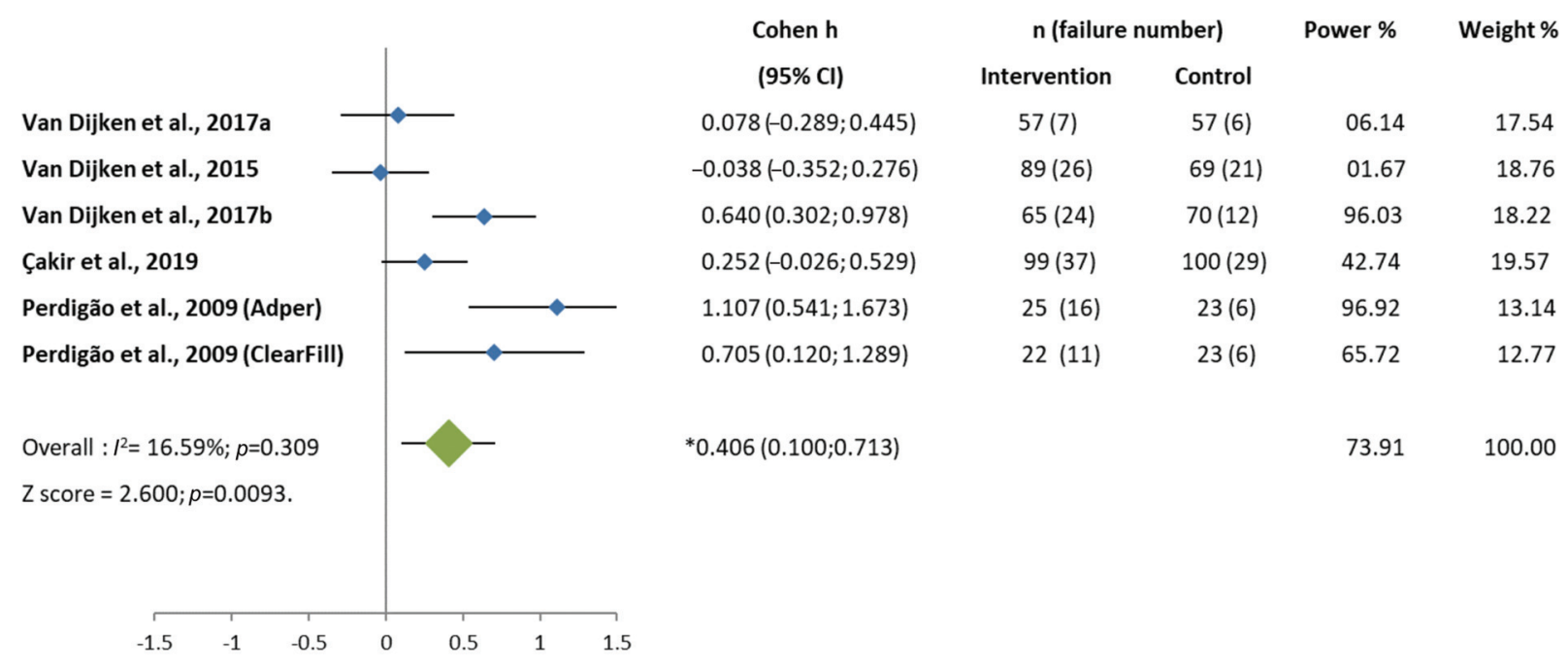

Negative values: favors self-etching adhesives; Positive values: favors etch-and-rise adhesives.

*Weights are from random effects model.

Fig. 2 Results of meta-analyses: Negative Cohen $\mathrm{H}$ values favor self-etching adhesives. Positive Cohen $\mathrm{H}$ values favor etch-and-rise adhesives. 

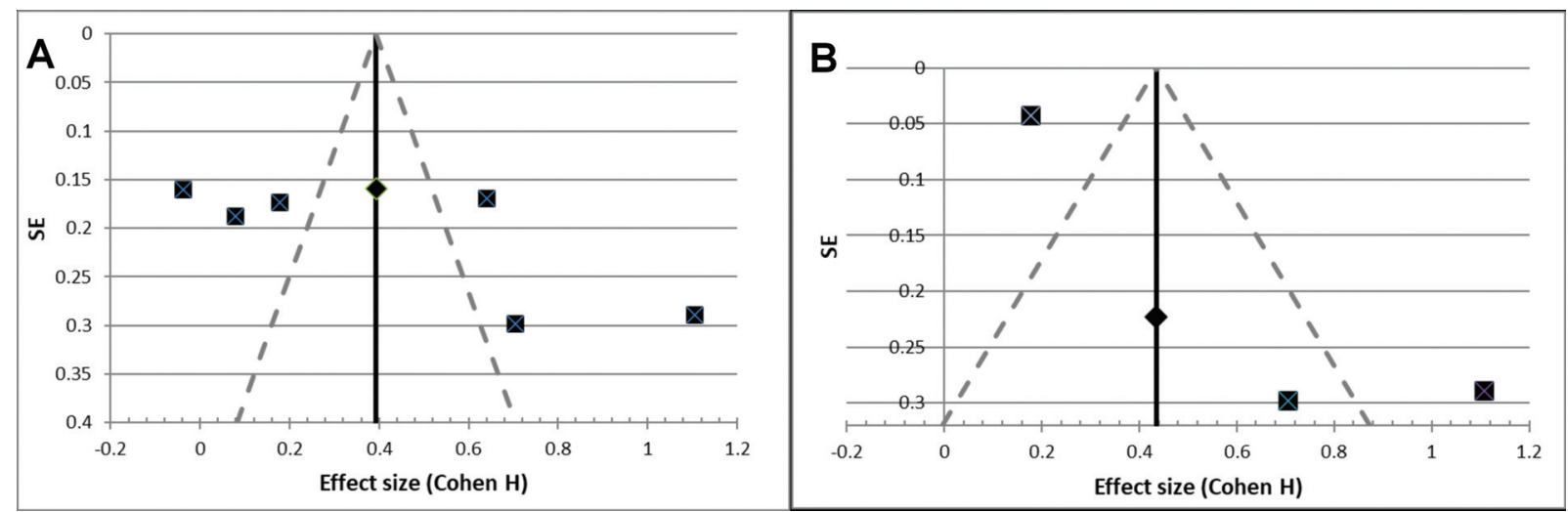

Fig. 3 Funnel plot (Cohen $\mathrm{H}$ effect size against standard errors) of publication bias, for all studies.

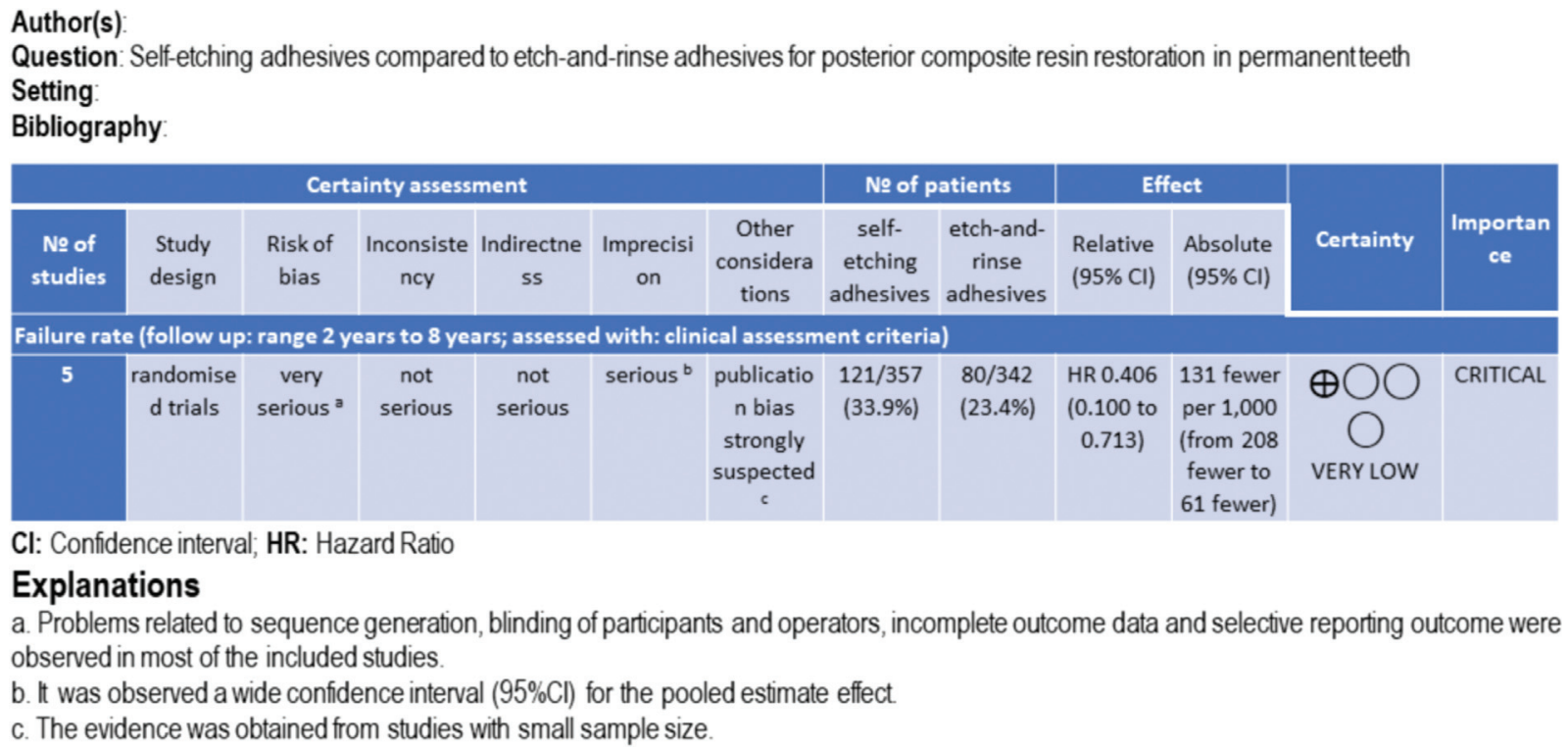

Fig. 4 Grades of Recommendations, Assessment, Development and Evaluation (GRADE).

required to yield reasonable standard errors. ${ }^{10,11}$ When interpreting the high p-value and the wide $95 \% \mathrm{CI}$ found for the meta-analysis of all studies (with and without rubber dam), one must consider that the probability within the $\mathrm{Cl}$ is maximum at the point estimate (effect size of 0.403 , favoring etch-and-rinse adhesives) and decreases towards both upper (0.703) and lower (0.100) limits. The words "were included" should be removed. ${ }^{11}$ More specifically, the wide $\mathrm{CI}$ is the result of small sample sizes in the individual studies and can be improved by further studies with larger sample sizes. The presence of important publication bias (-Fig. 3) further supports the idea that the pooled studies have high variability.

The failures in marginal adaptation and marginal staining are closely related to the location of the dentine adhesive in the restoration, supporting the interpretation that the failure rate was mostly related to the adhesive type used. Selfetching adhesives face a coupled diffusion challenge: the outward diffusion of dissolved mineral ions (due to acid etching) and the inward diffusion of both the primer and the bonding molecules, with embedding of dissolved calcium phosphates within the dentin hybrid layer would destabilize the adhesive interface with time. ${ }^{27}$ The lack of intermediate step potentially includes difficulties for establishing a reasonable hybrid layer, and the current recommendation includes a separate selective enamel acid conditioning prior to applying self-etching adhesives. ${ }^{27}$ Such selective enamel conditioning was not used in any of the studies included in the current meta-analysis.

Our results are consistent with previous meta-analyses indicating higher sensitivity of self-etching adhesives to long-term water storage in vitro ${ }^{28}$ and higher annual failure rates of one step self-etching adhesives in non-cervical carious lesions compared to both two steps etch-and-rinse and two steps self-etching adhesives. ${ }^{29}$

The thinner hybrid layer obtained with self-etch adhesives $^{4}$ is another probable explanation for the higher failure rate of resin composite restoration in posterior teeth using self-etch adhesives.

In order to contribute to the planning of future longitudinal studies on the failure rates of etch-and-rinse versus onestep adhesives in posterior composite restorations, the use of rubber dam in paired groups recommended. For sample size calculations, to the best of available evidence identified in the 
current meta-analysis, it would be recommended the use of an effect size Cohen $\mathrm{H}$ of 0.406 (close to the cut-off of 5 , for medium effect size), a two-tailed $5 \%$ significance level, power of $80 \%$, which would result in a sample size of 48 per group. This estimation does not include any sample size loss due to the failure in compliance with study recall appointments during the follow-up period.

\section{Conclusion}

In conclusion, current available evidence indicates that etchand rinse adhesives performed better than self-etching adhesives in terms of failure rates in posterior composite restorations. But the certainty of evidence is very low, indicating the necessity of more well-conducted studies with larger sample sizes and less risk of bias. Improved ad hoc planning for future studies is required to achieve scientific evidence with smaller variability.

\section{Conflict of Interest}

None declared.

\section{Acknowledgments}

PhD scholarships for B.R.V. and E.L.A.D. funded by CNPq (Brazilian Ministry of Science, Technology, and Innovation) are greatly acknowledged.

\section{References}

1 Sofan E, Sofan A, Palaia G, Tenore G, Romeo U, Migliau G. Classification review of dental adhesive systems: from the IV generation to the universal type. Ann Stomatol (Roma) 2017;8 (01):1-17

2 Häfer M, Schneider H, Rupf S, et al. Experimental and clinical evaluation of a self-etching and an etch-and-rinse adhesive system. J Adhes Dent 2013;15(03):275-286

3 Giannini M, Makishi P, Ayres AP, et al. Self-etch adhesive systems: a literature review. Braz Dent J 2015;26(01):3-10

4 Tay FR, King NM, Chan KM, Pashley DH. How can nanoleakage occur in self-etching adhesive systems that demineralize and infiltrate simultaneously? J Adhes Dent 2002;4(04):255-269

5 Correa MB, Peres MA, Peres KG, Horta BL, Barros AD, Demarco FF. Amalgam or composite resin? Factors influencing the choice of restorative material. J Dent 2012;40(09):703-710

6 Demarco FF, Collares K, Correa MB, Cenci MS, Moraes RR, Opdam NJ. Should my composite restorations last forever? Why are they failing?. Braz Oral Res 2017;31(Suppl 1):e56

7 Kubo S, Kawasaki A, Hayashi Y. Factors associated with the longevity of resin composite restorations. Dent Mater J 2011;30 (03):374-383

8 van Dijken JW, Pallesen U. Four-year clinical evaluation of Class II nano-hybrid resin composite restorations bonded with a one-step self-etch and a two-step etch-and-rinse adhesive. J Dent 2011;39 (01):16-25

9 van Dijken JW, Pallesen U. Eight-year randomized clinical evaluation of Class II nanohybrid resin composite restorations bonded with a one-step self-etch or a two-step etch-and-rinse adhesive. Clin Oral Investig 2015;19(06):1371-1379

10 Greenland S, Senn SJ, Rothman KJ, et al. Statistical tests, P values, confidence intervals, and power: a guide to misinterpretations. Eur J Epidemiol 2016;31(04):337-350

11 Szklo M, Nieto J. Epidemiology: Beyond the Basic. 4th ed. Burlington: Jones \& Bartlett Publishers; 2019

12 Page MJ, McKenzie JE, Bossuyt PM, et al. The PRISMA 2020 statement: an updated guideline for reporting systematic reviews. BMJ 2021;372:n71

13 Maia LC, Antonio AG. Systematic reviews in dental research. A guideline. J Clin Pediatr Dent 2012;37(02):117-124

14 Higgins JP, Altman DG, Gøtzsche PC, et al. The Cochrane Collaboration's tool for assessing risk of bias in randomised trials. BMJ 2011;343:d5928

15 Cohen J. Statistical Power Analysis for the Behavioral Sciences. Hillsdale: Lawrence Erlbaum Associates; 1998

16 Sánchez-Meca J, Marín-Martínez F, Chacón-Moscoso S. Effect-size indices for dichotomized outcomes in meta-analysis. Psychol Methods 2003;8(04):448-467

17 Boslaught S. Statistics in a Nutshell-A Desktop Quick Reference. O'Reilly2nd ed.; 2011

18 Neyeloff JL, Fuchs SC, Moreira LB. Meta-analyses and Forest plots using a Microsoft excel spreadsheet: step-by-step guide focusing on descriptive data analysis. BMC Res Notes 2012;5(01):52

19 Higgins JP, Thompson SG, Deeks JJ, Altman DG. Measuring inconsistency in meta-analyses. BMJ 2003;327(7414):557-560

20 Hedges LV, Pigott TD. The power of statistical tests in metaanalysis. Psychol Methods 2001;6(03):203-217

21 Sterne JA, Egger M. Funnel plots for detecting bias in metaanalysis: guidelines on choice of axis. J Clin Epidemiol 2001;54 (10):1046-1055

22 Ryan R, Hill S. How to GRADE the quality of the evidence. Cochrane Consumers and Communication Group. Version 3.0 December 2016. Accessed on July 1, 2021 at: http://cccrg. cochrane.org/author-resources

23 Perdigão J, Dutra-Corrêa M, Anauate-Netto C, et al. Two-year clinical evaluation of self-etching adhesives in posterior restorations. J Adhes Dent 2009;11(02):149-159

24 van Dijken JW, Pallesen U. Three-year randomized clinical study of a one-step universal adhesive and a two-step self-etch adhesive in class II composite restorations. J Adhes Dent 2017;19(04): 287-294

25 van Dijken JWV, Pallesen U. Durability of a low shrinkage TEGDMA/HEMA-free resin composite system in Class II restorations. A 6-year follow up. Dent Mater 2017;33(08):944-953

26 Çakır NN, Demirbuga S. The effect of five different universal adhesives on the clinical success of class I restorations: 24-month clinical follow-up. Clin Oral Investig 2019;23(06):2767-2776

27 Van Meerbeek B, Yoshihara K, Van Landuyt K, Yoshida Y, Peumans M. From Buonocore's pioneering acid-etch technique to selfadhering restoratives. a status perspective of rapidly advancing dental adhesive technology. J Adhes Dent 2020;22(01):7-34

28 De Munck J, Mine A, Poitevin A, et al. Meta-analytical review of parameters involved in dentin bonding. J Dent Res 2012;91(04): 351-357

29 Peumans M, De Munck J, Mine A, Van Meerbeek B. Clinical effectiveness of contemporary adhesives for the restoration of non-carious cervical lesions. A systematic review. Dent Mater 2014;30(10):1089-1103 